\title{
Role of deformation in the nonmesonic decay of light hypernuclei
}

\author{
K. Hagino* and A. Parreño ${ }^{\dagger}$ \\ Institute for Nuclear Theory, Department of Physics, University of Washington, Seattle, Washington 98195
}

(Received 28 April 2000; published 19 March 2001)

\begin{abstract}
We discuss the nonmesonic decay of deformed $p$-shell hypernuclei. The Nilsson model with angular momentum projection is employed in order to take into account the deformation effects. The nonmesonic decay rate and the intrinsic $\Lambda$ asymmetry parameter decrease as a function of the deformation parameter, while the ratio of the neutron- to proton-induced decay rates increases. We find that the deformation effects change these observables by about $10 \%$ for ${ }_{\Lambda}^{9} \mathrm{Be}$ from the spherical limit.
\end{abstract}

DOI: 10.1103/PhysRevC.63.044318 PACS number(s): 21.80.+a, 21.60.Cs, 13.75.Ev, 25.80.Pw

\section{INTRODUCTION}

One of the main issues of nuclear physics is to understand the nucleon-nucleon $(N N)$ interaction. The $|\Delta S|=1 N N$ interaction is particularly important in this respect, since the change of strangeness can be used as a signature to study both the parity-conserving (PC) and the parity-violating (PV) amplitudes. This is in clear contrast to the $\Delta S=0$ weak $N N$ interaction, where the weak PC signal is masked by the strong interaction.

Due to the lack of stable $\Lambda$-particle beams, the weak decay of $\Lambda$ hypernuclei has been the only source of information on the weak four-baryon $|\Delta S|=1$ interaction. Single $\Lambda$-hypernuclei are typically produced via either hadronic reactions, as $\left(K^{-}, \pi^{-}\right)[1,2]$ or $\left(\pi^{+}, K^{+}\right)$[3], or electroproduction mechanisms, as $\left(e, e^{\prime} K^{+}\right)$[4]. These hypernuclei are typically produced in an excited state and reach their ground state by electromagnetic $\gamma$ and/or particle emission. Once they are stable against strong decay, they decay via weak interaction mechanisms that are nonleptonic in nature and violate isospin, parity, and strangeness. Since the mesonic decay mode, $\Lambda \rightarrow \pi N$, is Pauli blocked in the nuclear medium, hypernuclei with $A \gtrsim 5$ predominantly decay through the nonmesonic decay (NMD) mode, $\Lambda N \rightarrow N N$.

In order to learn about the weak $\Lambda N \rightarrow N N$ interaction from the theoretical side, one has to take into account different inputs as accurately as possible. These include the description of nuclear structure, the choice of the strong baryon-baryon $(B B)$ potential model $[5,6], \Delta I=1 / 2$ violations [7] and the importance of the $3 N$ emission channel, $\Lambda n p \rightarrow n n p$ [8,9]. In Refs. [10,11], a one-meson-exchange (OME) model was applied to calculate the nonmesonic decay observables of the $p$-shell ${ }_{\Lambda}^{11} \mathrm{~B}$ and ${ }_{\Lambda}^{12} \mathrm{C}$ and the $s$-shell ${ }_{\Lambda}^{5} \mathrm{He}$ and ${ }_{\Lambda}^{3} \mathrm{H}$ hypernuclei. We included the virtual exchange of the ground-state pseudoscalar and vector mesons $\rho, \eta, \omega$, $K$, and $K^{*}$, in addition to the long-ranged pion. Except for the hypertriton, where the hypernuclear wave function was calculated exactly using the Faddeev formalism, the structure

\footnotetext{
*Present address: Yukawa Institute for Theoretical Physics, Kyoto University, Kyoto 606-8502, Japan.

${ }^{\dagger}$ Present address: Department Estructura i Constituents de la Materia, Facultat de Fisica, Diagonal 647, E-08028 Barcelona, Spain.
}

of the initial hypernucleus was described in a shell-model framework that assumed spherical configuration. In these calculations, the strong $B B$ interaction was accounted for using the Nijmegen $B B$ potential model [5]. Monopole form factors at each vertex were included in order to regularize the weak potential, while the weak baryon-baryon-meson coupling constants were derived based on $\mathrm{SU}_{w}(6)$ and softmeson theorems. The total NMD rate and the asymmetry in the distribution of emitted protons from the decay of polarized hypernuclei were in good agreement with the experimental data. However, the theoretical values for the neutronto-proton ratio were found to be very small compared to the experimental data. Several attempts have been made to reconcile this discrepancy [7-9,12-14], but none of them has solved this problem yet.

Our aim in this paper is to investigate how much these observables depend on the deformation of hypernuclei. All previous calculations were performed using the spherical configuration with no mixing, however, it is well known that many $p$-shell nuclei are deformed in the ground state. For instance, the quadrupole deformation parameter extracted from the experimental quadrupole moment [15] is $\beta_{2}=0.65$ for ${ }^{10} \mathrm{~B}$ and -0.71 for ${ }^{11} \mathrm{C}$. It may be important to take these deformation effects into account in order to describe quantitatively the nonmesonic decay of $p$-shell hypernuclei. Deformed hypernuclei can be described using several models such as the $\alpha$-cluster model [16] or the deformed selfconsistent Hartree-Fock method. In fact, one can also use realistic wave functions obtained by a diagonalization of a shell-model Hamiltonian for $p$-shell nuclei, as in Ref. [17]. In the present paper, however, in order to perform a systematic study, we use instead the Nilsson model $[18,19]$ as a simplified Hartree-Fock method.

The paper is organized as follows. In Sec. II, we present the relevant formulas to evaluate the NMD observables in a OME model. In Sec. III, we briefly review the deformed shell model based on the Nilsson model. Section IV presents the deformation dependence of the nonmesonic observables for the decay of ${ }_{\Lambda}^{9} \mathrm{Be}$, whose ${ }^{8} \mathrm{Be}$ core is known to be largely deformed. Although there are no experimental data for this hypernucleus at present, we choose this system as the simplest nonspherical $p$-shell hypernucleus and as a representative example of deformed $p$-shell nuclei. We compare our theoretical predictions with the typical experimental data for other $p$-shell hypernuclei. Section V summarizes the paper. 


\section{NONMESONIC WEAK DECAY IN A ONE-MESON- EXCHANGE MODEL}

Assuming that the initial hypernucleus is at rest, the NMD rate is

$$
\begin{aligned}
\Gamma_{\mathrm{nm}}= & \int \frac{d^{3} k_{1}}{(2 \pi)^{3}} \int \frac{d^{3} k_{2}}{(2 \pi)^{3}} \sum_{\substack{M_{I}\{R\} \\
\{1\}\{2\}}}(2 \pi) \delta\left(M_{H}-E_{R}-E_{1}-E_{2}\right) \\
& \times \frac{1}{(2 J+1)}\left|\mathcal{M}_{f i}\right|^{2}
\end{aligned}
$$

where $\mathcal{M}_{f i}$ is the hypernuclear transition amplitude. The quantities $M_{H}, E_{R}, E_{1}$, and $E_{2}$ are the mass of the hypernucleus, the energy of the residual $(A-2)$-particle system, and the total asymptotic energies of the emitted nucleons, respectively. The integration variables $\vec{k}_{1}$ and $\vec{k}_{2}$ are the momenta of the two baryons in the final state. The momentumconserving $\delta$ function has been used to integrate over the momentum of the residual nucleus. The sum, together with the factor $1 /(2 J+1)$, indicates an average over the initial hypernucleus spin projections, $M_{I}$, and a sum over all quantum numbers of the residual $(A-2)$-particle system, $\{R\}$, as well as the spin and isospin projections of the exiting particles, $\{1\}$ and $\{2\}$. In general, one can write the total nonmesonic decay rate as $\Gamma_{\mathrm{nm}}=\Gamma^{\Lambda N \rightarrow N N}=\Gamma_{n}+\Gamma_{p}$, where $\Gamma_{n}$ $(\Lambda n \rightarrow n n)$ stands for the neutron-induced decay and $\Gamma_{p}$ $(\Lambda p \rightarrow n p)$ for the proton-induced one.

In addition to the total and partial decay rates, we also calculate the intrinsic $\Lambda$ asymmetry parameter. When working with polarized hypernuclei and in combination with coincidence measurements of the decay particles, one can study the angular distribution of particles coming from the $\Lambda N$ $\rightarrow N N$ weak decay. Due to the interference between the PV and PC amplitudes, the distribution of the emitted protons in the weak decay displays an angular asymmetry with respect to the polarization axis. The asymmetry $\mathcal{A}$, defined by

$$
\mathcal{A}=P_{y} \frac{3}{J+1} \frac{\operatorname{Tr}\left(\mathcal{M}_{f i} S_{y} \mathcal{M}_{f i}^{\dagger}\right)}{\operatorname{Tr}\left(\mathcal{M}_{f i} \mathcal{M}_{f i}^{\dagger}\right)}
$$

is expressed in terms of the hypernuclear polarization created in the strong production reaction, $P_{y}$, the $J$-spin operator along the polarization axis, $S_{y}$, and the total spin of the initial hypernucleus, $J$. In Ref. [20] it is shown that the asymmetry follows a simple $\cos \chi$ dependence, i.e., $\mathcal{A}$ $=P_{y} A_{p} \cos \chi$, where $\chi$ stands for the angle between the direction of the proton and the polarization axis. The hypernuclear asymmetry parameter $A_{p}$ is characteristic of the hypernuclear weak decay process and depends on $J$ and the intensity of protons exiting along the quantization axis for the different spin projections of the hypernucleus. At $\chi$ $=0^{\circ}$, the asymmetry in the distribution of protons is thus determined by the product $\mathcal{A}=P_{y} A_{p}$. In the following, we assume a weak coupling scheme where the $\Lambda$ hyperon is coupled only to the ground state of the $(A-1)$-particle core. In this scheme, simple angular momentum algebra relates the hypernuclear polarization $P_{y}$ to the $\Lambda$ polarization $p_{\Lambda}$ and
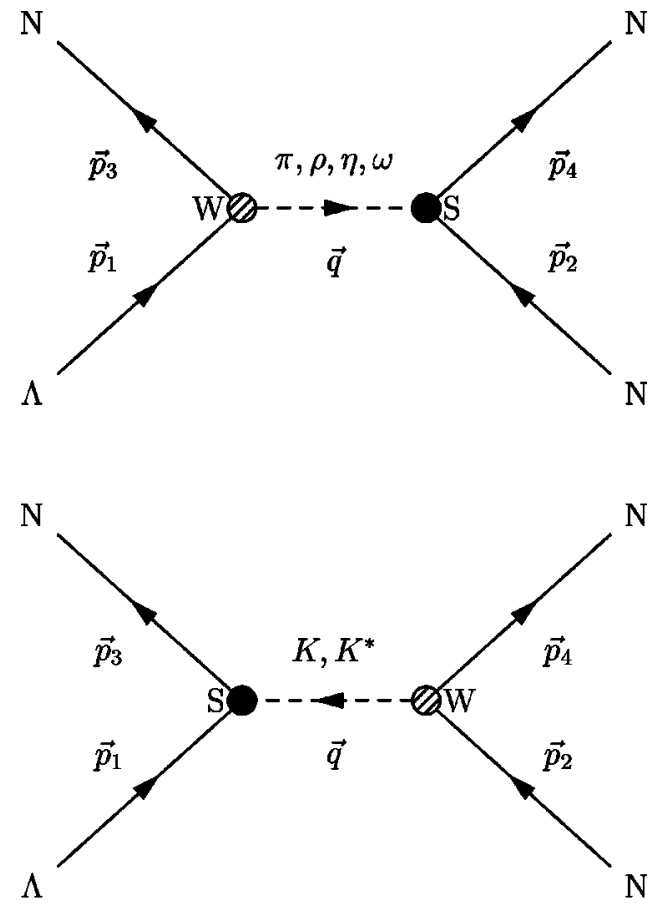

FIG. 1. Free Feynman diagrams for the $\Lambda N \rightarrow N N$ transition mediated by the exchange of the nonstrange $\pi, \eta, \rho, \omega$ (left) and strange $K, K^{*}$ (right) mesons. The shaded circle (filled) stands for the weak (strong) vertex.

relates the hypernuclear asymmetry parameter $A_{p}$ to the intrinsic $\Lambda$ asymmetry parameter $a_{\Lambda}$, such that $\mathcal{A}=p_{\Lambda} a_{\Lambda}$ $=P_{y} A_{p}$.

The nonmesonic decay of hypernuclei proceeds through a two-body mechanism. Therefore in order to evaluate the transition amplitude in Eq. (1), one has to decompose the $(A-1)$-core wave function into a set of states in which a nucleon couples to the residual $(A-2)$-particle state. This can be done using the coefficients of fractional parentage (CFP), which are defined by

$$
\left|J M, T T_{z}\right\rangle=\sum_{J_{R}, T_{R}, j}\left\langleJ T \left\{\left|J_{R} T_{R}, j t\right\rangle\left[\left|J_{R} T_{R}\right\rangle \otimes|j t\rangle\right]_{J M, T T_{z}}\right.\right.
$$

where $J_{R}$ and $T_{R}$ are the spin and isospin of the residual nucleus. The weak potential responsible for this transition can be obtained by making a nonrelativistic reduction of the free Feynman amplitude depicted in Fig. 1. In Table I we show the strong and weak Hamiltonians for pseudoscalar (PS) and vector (V) mesons. $A, B, \alpha, \beta$, and $\epsilon$ stand for the appropriate baryon-baryon-meson weak coupling constants, while $g\left(g^{\mathrm{V}}, g^{\mathrm{T}}\right)$ represents the strong (vector, tensor) coupling. Details of the derivation of the transition potential can be found in Ref. [10] and here only the final expression will be presented. For pseudoscalar mesons, the potential is

$$
V_{p s}(\vec{q})=-G_{F} m_{\pi}^{2} \frac{g}{2 M}\left(\hat{A}+\frac{\hat{B}}{2 \bar{M}} \vec{\sigma}_{1} \cdot \vec{q}\right) \frac{\vec{\sigma}_{2} \cdot \vec{q}}{\vec{q}^{2}+\mu^{2}}
$$


TABLE I. Weak and strong Hamiltonians for pseudoscalar (PS) and vector $(\mathrm{V})$ meson exchanges. The weak Hamiltonians are in units of $G_{F} m_{\pi}^{2}$ and $\Psi(\Phi)$ stands for the baryon (meson) field.

\begin{tabular}{ccc}
\hline \hline & PS & $\mathrm{V}$ \\
\hline Strong & $i g \bar{\Psi} \gamma_{5} \Psi \phi$ & $\bar{\Psi}\left[g^{\mathrm{V}} \gamma^{\mu}+i \frac{g^{\mathrm{T}}}{2 M} \sigma^{\mu \nu} q_{\nu}\right] \Psi \phi$ \\
Weak & $i \bar{\Psi}\left(A+B \gamma_{5}\right) \Psi \phi$ & $\bar{\Psi}\left[\alpha \gamma^{\mu}-\beta i \frac{\sigma^{\mu \nu} q_{\nu}}{2 \bar{M}}+\varepsilon \gamma^{\mu} \gamma_{5}\right] \Psi \phi$ \\
\hline \hline
\end{tabular}

where $G_{F} m_{\pi}^{2}=2.21 \times 10^{-7}$ is the Fermi coupling constant, $\vec{q}$ is the momentum carried by the meson directed towards the strong vertex, $\mu$ is the meson mass, and $M(\bar{M})$ is the average of the baryon masses at the strong (weak) vertex (and vice versa for the exchange of strange mesons). For vector mesons, the potential is

$$
\begin{aligned}
V_{v}(\vec{q})= & G_{F} m_{\pi}^{2}\left(g^{\mathrm{V}} \hat{\alpha}-\frac{(\hat{\alpha}+\hat{\beta})\left(g^{\mathrm{V}}+g^{\mathrm{T}}\right)}{4 M \bar{M}}\left(\vec{\sigma}_{1} \times \vec{q}\right) \cdot\left(\vec{\sigma}_{2} \times \vec{q}\right)\right. \\
& \left.-\mathrm{i} \frac{\hat{\varepsilon}\left(g^{\mathrm{V}}+g^{\mathrm{T}}\right)}{2 M}\left(\vec{\sigma}_{1} \times \vec{\sigma}_{2}\right) \cdot \vec{q}\right) \frac{1}{\vec{q}^{2}+\mu^{2}} .
\end{aligned}
$$

The values of the strong and weak couplings are listed in Table III of Ref. [10]. In Eqs. (4) and (5) the operators $\hat{A}, \hat{B}$, $\hat{\alpha}, \hat{\beta}$, and $\hat{\varepsilon}$ contain, apart from the weak coupling constants, the specific isospin dependence of the potential, which is $\vec{\tau}_{1} \cdot \vec{\tau}_{2}$ for the isovector $\pi$ and $\rho$ mesons, $\hat{1}$ for the isoscalar $\eta$ and $\omega$ mesons, and a combination of both operators for the isodoublet $K$ and $K^{*}$. In order to derive Eqs. (4) and (5) we assumed the validity of the $\Delta I=1 / 2$ rule, which is known to experimentally dominate the decay of $\Lambda$ 's into pions. $\Delta I$ $=3 / 2$ transitions for vector mesons $\left(\rho\right.$ and $K^{*}$ ) are easily accommodated [7] in the formalism, and the results we present here account for such $\Delta I=1 / 2$ violations.

We obtain a regularized potential by including a monopole form factor at each vertex, $F\left(\vec{q}^{2}\right)=\left(\Lambda^{2}-\mu^{2}\right) /\left(\Lambda^{2}\right.$ $+\vec{q}^{2}$ ), where the value of the cutoff, $\Lambda$, different for each meson, is taken from the Jülich hyperon-nucleon interaction [6]. To incorporate the effects of the strong $N N$ interaction, we solve a $T$-matrix scattering equation in momentum space for the outgoing nucleons using the Nijmegen [5] potential models. For the initial bound two-baryon system we use a spin independent parametrization of the type

$$
f(r)=\left(1-e^{-r^{2} / a^{2}}\right)^{n}+b r^{2} e^{-r^{2} / c^{2}},
$$

with $a=0.5 \mathrm{fm}, b=0.25 \mathrm{fm}^{-2}, c=1.28 \mathrm{fm}$, and $n=2$. The results obtained with this parametrization [21] lay between those obtained with a microscopic finite-nucleus $G$-matrix calculation [22] using the soft-core and hard-core Nijmegen [23] models.

\section{DEFORMED SHELL MODEL}

As we mentioned in the previous section, we use a weak coupling scheme for the $\Lambda$ hyperon in the initial hypernucleus. To this end, we must describe the ground state of the core nucleus which may be deformed. The Nilsson model provides a simple and convenient framework to describe deformed nuclei, and has been widely used in the literature $[18,19,24-27]$. It is based on an anisotropic harmonic oscillator and its Hamiltonian reads

$$
\begin{aligned}
H= & H_{0}-\frac{4}{3} \sqrt{\frac{\pi}{5}} \delta m \omega_{0}^{2} Y_{20}(\theta) \\
= & -\frac{\hbar^{2}}{2 m} \nabla^{2}+\frac{1}{2} m \omega_{0}^{2} r^{2}+C \vec{l} \cdot \vec{s}+D\left(\vec{l}^{2}-\left\langle\vec{l}^{2}\right\rangle_{N}\right) \\
& -\frac{4}{3} \sqrt{\frac{\pi}{5}} \delta m \omega_{0}^{2} Y_{20}(\theta) .
\end{aligned}
$$

Here $\delta$ is a deformation parameter, $\vec{l}$ and $\vec{s}$ are the singleparticle orbital and the spin angular momenta, and $C$ and $D$ are adjustable parameters. $\left\langle\vec{l}^{2}\right\rangle_{N}=N(N+3) / 2$ is the expectation value of $\vec{l}^{2}$ averaged over one major shell with quantum number $N$. The relation between $\delta$ and $\beta_{2}$ is given by [28]

$$
\beta_{2}=\frac{4}{3} \sqrt{\frac{\pi}{5}} \frac{\delta}{1-2 \delta / 3} .
$$

Since the Nilsson Hamiltonian (8) violates rotational invariance, the total angular momentum $\vec{j}=\vec{l}+\vec{s}$ is not a good quantum number. However, the projection of $\vec{j}$ onto the $z$ direction, $k$, is conserved, and the single-particle levels are characterized by $k$ and other quantum numbers. We expand a Nilsson single-particle level, $\psi_{k(q)}$, in terms of the eigenfunctions of the spherical harmonic oscillator Hamiltonian $H_{0}, \phi_{n l j k}$, as

$$
\psi_{k(q)}=\sum_{n l j} x_{n l j k}^{(q)} \phi_{n l j k}
$$

where $q$ are quantum numbers other than $k$. We choose $x_{n l j-k}^{(q)}=(-1)^{j-k} x_{n l j k}^{(q)}$ so that the eigenvalues of the Nilsson Hamiltonian do not depend on the sign of the projection of total angular momentum [27]. We denote the creation operator of $\psi_{k}$ as $a_{k}^{\dagger}$ and that of $\phi_{j k}$ as $b_{j k}^{\dagger}$. We explicitly express only the $j$ and $k$ quantum numbers to simplify the notation. Intrinsic wave functions, i.e., eigenfunctions of the Nilsson Hamiltonian (8), are given by

$$
\left|\chi_{K}\right\rangle=a_{k_{1}}^{\dagger} a_{k_{2}}^{\dagger} \cdots a_{k_{n}}^{\dagger}|0\rangle=\prod_{i=1}^{n}\left(\sum_{j} x_{j k_{i}} b_{j k_{i}}^{\dagger}\right)|0\rangle,
$$

where the $K$ quantum number is the sum over all $k_{i}$. The intrinsic wave function (11) is not an eigenstate of the total angular momentum $\vec{J}$, and thus has to be projected out to a good angular momentum state. This can be achieved by using the projector given by $[19,24,25]$ 


$$
\hat{P}_{M K}^{J}=\frac{2 J+1}{8 \pi^{2}} \int d \Omega D_{M K}^{J}(\Omega) \hat{R}(\Omega),
$$

where $\Omega$ are Euler angles and $D_{M K}^{J}(\Omega)$ and $\hat{R}(\Omega)$ are the Wigner $D$ function and the rotation operator, respectively.

For systems with a single Nilsson level, such as ${ }^{8} \mathrm{Be}$ which we discuss in the next section, the CFP can be analytically obtained [26,27]. Note that a single Nilsson level can accommodate up to four nucleons, i.e., two protons and two neutrons. For three-particle systems (two neutrons and one proton, for example), the wave function is given by

$$
\begin{aligned}
\Psi_{J M}= & {\left[N(3)_{J}\right]^{-1} \hat{P}_{M, K=k}^{J} a_{k \nu}^{\dagger} a_{-k \nu}^{\dagger} a_{k \pi}^{\dagger}|0\rangle } \\
= & {\left[N(3)_{J}\right]^{-1} \sum_{j_{1}, j_{2}, j_{3}} \sum_{J_{12}} x_{j_{1} k} x_{j_{2}-k} x_{j_{3} k} } \\
& \times\left\langle j_{1} k j_{2}-k \mid J_{12} 0\right\rangle\left\langle J_{12} 0 j_{3} k \mid J k\right\rangle \\
& \times\left(\left[a_{j_{1} \nu}^{\dagger} a_{j_{2} \nu}^{\dagger}\right]_{J_{12}} a_{j_{3}}^{\dagger}\right)_{J M}|0\rangle,
\end{aligned}
$$

where $\pi$ stands for proton and $\nu$ for neutron. The normalization factor $N(3)_{J}$ is given by [26]

$$
\left[N(3)_{J}\right]^{2}=\sum_{j, J_{12}} \delta_{J_{12}, \text { even }}\left(x_{j k}\right)^{2} U\left(J_{12}, k\right)\left\langle J_{12} 0 j k \mid J k\right\rangle^{2},
$$

where

$$
U(J, k)=2 \sum_{j_{1}, j_{2}}\left(x_{j_{1} k}\right)^{2}\left(x_{j_{2} k}\right)^{2}\left\langle j_{1} k j_{2}-k \mid J 0\right\rangle .
$$

The isospin of this system is $1 / 2$. For four-nucleon systems, the wave function reads

$$
\begin{aligned}
\Psi_{J M}= & {\left[N(4)_{J}\right]^{-1} \hat{P}_{M, K=0}^{J} a_{k \nu}^{\dagger} a_{-k \nu}^{\dagger} a_{k \pi}^{\dagger} a_{-k \pi}^{\dagger}|0\rangle } \\
= & {\left[N(4)_{J}\right]^{-1} \sum_{j_{1}, j_{2}} \sum_{j_{3}, j_{4}} \sum_{J_{12}, J_{R}} x_{j_{1} k} x_{j_{2}-k} x_{j_{3} k} x_{j_{4}-k} } \\
& \times\left\langle j_{1} k j_{2}-k \mid J_{12} 0\right\rangle\left\langle J_{12} 0 j_{3} k \mid J_{R} k\right\rangle\left\langle J_{R} k j_{4}-k \mid J 0\right\rangle \\
& \times\left\{\left[\left(a_{j_{1} \nu}^{\dagger} a_{j_{2} \nu}^{\dagger}\right)_{J_{12}} a_{j_{3} \pi}^{\dagger}\right]_{J_{R}} a_{j_{4} \pi}^{\dagger}\right\}_{J M}|0\rangle,
\end{aligned}
$$

with the normalization given by [26]

$$
\begin{aligned}
{\left[N(4)_{J}\right]^{2}=} & \sum_{J_{12}, J_{34}} \delta_{J_{12}, \text { even }} \delta_{J_{34}, \text { even }} U\left(J_{12}, k\right) U\left(J_{34}, k\right) \\
& \times\left\langle J_{12} 0 J_{34} 0 \mid J 0\right\rangle^{2}
\end{aligned}
$$

The isospin of this wave function is 0. Comparing Eqs. (14) and (18), the CFP for the four-particle system reads

$$
\mathcal{C}(j)=\left\langleJ T \left\{\left|J_{R} T_{R}, j t\right\rangle=-\sqrt{2} x_{j-k}\left\langle J_{R} k j-k \mid J 0\right\rangle \frac{N(3)_{J_{R}}}{N(4)_{J}} .\right.\right.
$$

\section{NONMESONIC DECAY OF ${ }_{\Lambda}^{9} \mathrm{Be}$}

Let us now apply the deformed shell model of Sec. III to the nonmesonic decay of ${ }_{\Lambda}^{9} \mathrm{Be}$. The quadrupole moment of the neighbor nucleus ${ }^{9} \mathrm{Be}$ was measured to be $5.86 e \mathrm{fm}^{2}$ [15], from which we extract the quadrupole deformation parameter $\beta_{2}=1.00$ using the radius parameter $r_{0}=1.2 \mathrm{fm}$. Several theoretical calculations suggest that the core nucleus ${ }^{8} \mathrm{Be}$ and the ${ }_{\Lambda}^{9} \mathrm{Be}$ hypernucleus also have similar deformation parameters with the same sign [16]. Our interest is to discuss such deformation effects on nonmesonic decay observables.

As is discussed in Sec. II, the use of the CFP allows us to write the hypernuclear transition amplitude $\mathcal{M}_{f i}$ in terms of elementary two-body amplitudes. Therefore, our first task is to compute these coefficients for the core nucleus ${ }^{8} \mathrm{Be}$. We assume the inert spherical ${ }^{4} \mathrm{He}$ core and explicitly work with only the four valence nucleons. Diagonalizing the Nilsson Hamiltonian (8), one finds that the lowest Nilsson level for the valence nucleons has $k=1 / 2$ for prolate deformation [19]. We diagonalize the Nilsson Hamiltonian in the $\Delta N$ $=0$ states. Contributions from the $\Delta N=2$ can be neglected unless the deformation is large. The $k=1 / 2$ state is thus

$$
\left|\psi_{k=1 / 2}\right\rangle=x\left|\phi_{p_{3 / 2,1 / 2}}\right\rangle+y\left|\phi_{p_{1 / 2,1 / 2}}\right\rangle
$$

where $x$ and $y$ are determined by diagonalizing the Nilsson Hamiltonian within this configuration space and depend upon the deformation of ${ }^{8} \mathrm{Be}$. Using Eq. (20), the CFP's are found to be

$$
\left[\mathcal{C}\left(p_{3 / 2}\right)\right]^{2}=\frac{3 x^{8}+3 x^{6} y^{2}+9 x^{4} y^{4}}{3 x^{8}+4 x^{6} y^{2}+18 x^{4} y^{4}+10 y^{8}}
$$

for the $p_{3 / 2}$ state, and

$$
\left[\mathcal{C}\left(p_{1 / 2}\right)\right]^{2}=\frac{x^{6} y^{2}+9 x^{4} y^{4}+10 y^{8}}{3 x^{8}+4 x^{6} y^{2}+18 x^{4} y^{4}+10 y^{8}}
$$

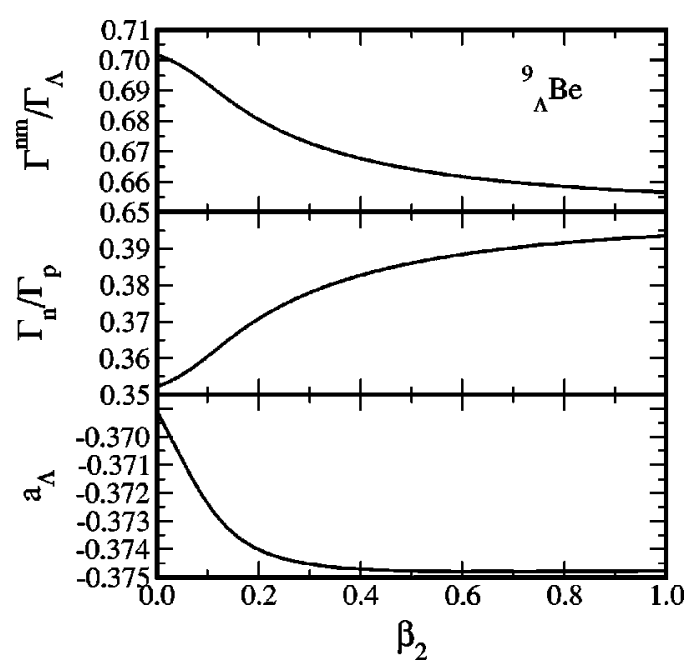

FIG. 2. The nonmesonic decay observables for ${ }_{\Lambda}^{9} \mathrm{Be}$ as a function of the deformation parameter $\beta_{2}$. 
TABLE II. Shell model spectroscopic factors of Cohen and Kurath [17] for pickup of $p$-shell nucleons in ${ }^{8} \mathrm{Be}$.

\begin{tabular}{lrccrc}
\hline \hline$n l j$ & Energy & SF & $n l j$ & Energy & SF \\
\hline $1 p \frac{1}{2}$ & 0.000 & 0.8940 & $1 p \frac{3}{2}$ & -1.979 & 3.1000 \\
$1 p \frac{1}{2}$ & 9.321 & 0.0027 & $1 p \frac{3}{2}$ & 9.066 & 0.0003 \\
$1 p \frac{1}{2}$ & 10.222 & 0.0000 & $1 p \frac{3}{2}$ & 9.929 & 0.0004 \\
$1 p \frac{1}{2}$ & 14.868 & 0.0002 & $1 p \frac{3}{2}$ & 12.734 & 0.0000 \\
$1 p \frac{1}{2}$ & 19.862 & 0.0001 & $1 p \frac{3}{2}$ & 14.726 & 0.0020 \\
\hline \hline
\end{tabular}

for the $p_{1 / 2}$ state. Note that $\left[\mathcal{C}\left(p_{3 / 2}\right)\right]^{2}+\left[\mathcal{C}\left(p_{1 / 2}\right)\right]^{2}=1$. In the spherical limit, $x=1$ and $y=0$, so the CFP's become $\left[\mathcal{C}\left(p_{3 / 2}\right)\right]^{2}=1$ and $\left[\mathcal{C}\left(p_{1 / 2}\right)\right]^{2}=0$. The CFP for the deeply bound $1 s_{1 / 2}$ state is just equal to 1 since ${ }^{4} \mathrm{He}$ is a spin-isospin saturated nucleus.

Our results for the nonmesonic decay rate, $\Gamma^{\mathrm{nm}}$, in units of the free $\Lambda$ decay rate, $\Gamma_{\Lambda}=3.8 \times 10^{9} \mathrm{~s}^{-1}$, the neutron-toproton ratio, $\Gamma_{n} / \Gamma_{p}$, and the $\Lambda$ asymmetry parameter, $a_{\Lambda}$, are shown in Fig. 2 as a function of the deformation parameter $\beta_{2}$. We use an oscillator length $b_{N}$ of $1.65 \mathrm{fm}$ for nucleons, so that the experimental root mean square radius of ${ }^{9} \mathrm{Be}$ is reproduced. Following Refs. $[18,19]$, the parameters $C$ and $D$ in the Nilsson Hamiltonian (8) are taken to be $-0.16 \hbar \omega_{0}$ and 0 , respectively. As for the oscillator length $b_{\Lambda}$ for the $1 s_{1 / 2}$ wave function of the $\Lambda$ hyperon, we estimate it to be $1.5 \mathrm{fm}$ in order to reproduce its binding energy in ${ }_{\Lambda}^{9} \mathrm{Be}(=6.71 \pm 0.04 \mathrm{MeV}[16])$. From the figure, we see that $\Gamma^{\mathrm{nm}} / \Gamma_{\Lambda}$ is a decreasing function of $\beta_{2}$, while $\Gamma_{n} / \Gamma_{p}$ and $a_{\Lambda}$ are increasing functions. As we have already mentioned, the deformation parameter of ${ }^{8} \mathrm{Be}$ is expected to be close to 1 . We notice that the nonmesonic decay observables are altered by about $10 \%$ from the spherical limit at $\beta_{2}=1$.

An important question is whether this effect is significant when comparing to the experimental data. We note that the typical experimental uncertainties for nonmesonic decay of $p$-shell hypernuclei are $7 \%-17.5 \%$ for the total decay rate $[29-31], 46.2 \%-84.2 \%$ for the neutron-to-proton ratio $[30,31]$, and $50 \%-1000 \%$ for the asymmetry [32]. These experimental uncertainties are much larger than the theoretical one originating from the deformation effects. Thus we conclude that the spherical approximation gives a good estimate of the nonmesonic decay of $p$-shell nuclei, at least within the present experimental precision.

Before we close this section, we would like to stress that our conclusion is not altered qualitatively even if more realistic wave functions are used instead of the present schematic ones. For instance, using the shell-model CFP of Cohen and Kurath [17] for the decay of ${ }_{\Lambda}^{9} \mathrm{Be}$ (see Table II) we obtain $\Gamma^{\mathrm{nm}} / \Gamma_{\Lambda}=0.65, \Gamma_{n} / \Gamma_{p}=0.40$ and $a_{\Lambda}=-0.37$. Those num- bers have to be compared with the spherical limit values of $0.70,0.35$, and -0.37 , respectively. The same type of calculation for ${ }_{\Lambda}^{12} \mathrm{C}$ gives $\Gamma^{\mathrm{nm}} / \Gamma_{\Lambda}=0.73$ and $\Gamma_{n} / \Gamma_{p}=0.27$, to be compared with the spherical values 0.71 and 0.32 , respectively. As we see, the amount of deviation of those observables with respect to the spherical limit is of the same order as that obtained here using the Nilsson model. ${ }^{1}$

\section{SUMMARY}

We have discussed the role of nuclear structure in the nonmesonic decay of $p$-shell hypernuclei, especially focusing on the effects of deformation. To this end, we have used the Nilsson model with explicit angular momentum projection. We have studied the nonmesonic decay of ${ }_{\Lambda}^{9} \mathrm{Be}$ as a typical example of deformed $p$-shell hypernuclei. We have shown that the deformation effects change the total NMD rate and the neutron-to-proton ratio by about $10 \%$ from the spherical limit, while the $\Lambda$ asymmetry parameter shows less sensitivity. Although this value is not negligible, it still is smaller than the present typical experimental uncertainty and smaller than other theoretical uncertainties, e.g., the effects of $\mathrm{SU}(3)$ symmetry breaking $[10,13]$ or $\Delta I=1 / 2$ violations [7]. This indicates that the existing discrepancies between the experimental and theoretical values of hypernuclear weak decay observables cannot be attributed solely to deviations from the spherical configuration and still remain an open question. New experiments are urged in order to reduce the large experimental error bars, which prevent any definite conclusion about the reliability of the theoretical models.

Our conclusions may not be the same for heavier hypernuclei such as ${ }_{\Lambda}^{238} \mathrm{U}[33,34]$. There are a lot of intruder states in such heavy deformed systems, unlike $p$-shell nuclei where there is only a few, or maybe zero, intruder states. Therefore, an interesting future work would be to discuss the nonmesonic decay of heavy hypernuclei including the deformation effects. For that purpose, the projected shell model developed in Refs. [24,25], which also uses the Nilsson model with angular momentum projection, would provide a useful tool to describe the structure of deformed hypernuclei.

\section{ACKNOWLEDGMENTS}

We thank David Brown and Amour Margarian for useful and illuminating discussions. This work was supported by the U.S. Department of Energy under Grant No. DE-FG0300-ER41132.

\footnotetext{
${ }^{1}$ Note that the behavior of the observables for ${ }_{\Lambda}^{12} \mathrm{C}$ and ${ }_{\Lambda}^{9} \mathrm{Be}$ is opposite, due partly to the fact that the residual ${ }^{11} \mathrm{C}$ is oblate while ${ }^{8} \mathrm{Be}$ is prolate.
}

[1] J. J. Szymanski et al., Phys. Rev. C 43, 849 (1991).

[2] R. Grace et al., Phys. Rev. Lett. 55, 1055 (1985).

[3] H. Park et al., Phys. Rev. C 61, 054004 (2000).

[4] O. K. Baker et al., Nucl. Phys. A639, 189c (1998).
[5] V. G. J. Stoks and Th. A. Rijken, Phys. Rev. C 59, 3009 (1999).

[6] B. Holzenkamp, K. Holinde, and J. Speth, Nucl. Phys. A500, 485 (1989). 
[7] A. Parreño, A. Ramos, C. Bennhold, and K. Maltman, Phys. Lett. B 435, 1 (1998).

[8] W. M. Alberico, A. De Pace, M. Ericson, and A. Molinari, Phys. Lett. B 256, 134 (1991).

[9] A. Ramos, M. J. Vicente-Vacas, and E. Oset, Phys. Rev. C 55, 735 (1997).

[10] A. Parreño, A. Ramos, and C. Bennhold, Phys. Rev. C 56, 339 (1997).

[11] J. Golak et al., Phys. Rev. C 55, 2196 (1997).

[12] J. F. Dubach, G. B. Feldman, B. R. Holstein, and L. de la Torre, Ann. Phys. (N.Y.) 249, 146 (1996); L. de la Torre, Ph.D. thesis, University of Massachusetts, 1982.

[13] M. J. Savage and R. P. Springer, Phys. Rev. C 53, 441 (1996); 54, 2786(E) (1996).

[14] K. Sasaki, T. Inoue, and M. Oka, Nucl. Phys. A669, 331 (2000); A678, 455 (2000).

[15] F. Ajzenberg-Selove, Nucl. Phys. A506, 1 (1990); A490, 1 (1988).

[16] H. Bandō, T. Motoba, and J. Žofka, Int. J. Mod. Phys. A 5, 4021 (1990).

[17] S. Cohen and D. Kurath, Nucl. Phys. A101, 1 (1967).

[18] C. Gustafson, I. L. Lamm, B. Nilsson, and S. G. Nilsson, Ark. Fys. 36, 613 (1967).

[19] P. Ring and P. Schuck, The Nuclear Many-Body Problem (Springer-Verlag, New York, 1980).
[20] A. Ramos, E. van Meijgaard, C. Bennhold, and B. K. Jennings, Nucl. Phys. A544, 703 (1992).

[21] A. Parreño, A. Ramos, C. Bennhold, and D. Halderson, in Dynamical Features of Nuclei and Finite Fermi Systems (World Scientific, Singapore, 1994), p. 318.

[22] D. Halderson, Phys. Rev. C 48, 581 (1993).

[23] M. N. Nagels, T. A. Rijken, and J. J. de Swart, Phys. Rev. D 15, 2547 (1977); P. M. M. Maessen, Th. A. Rijken, and J. J. de Swart, Phys. Rev. C 40, 2226 (1989).

[24] K. Hara and Y. Sun, Int. J. Mod. Phys. E 4, 637 (1995).

[25] Y. Sun and K. Hara, Comput. Phys. Commun. 104, 245 (1997).

[26] D. Kurath, Nuclear and Particle Physics (Benjamin, New York, 1968), p. 199.

[27] R. D. Lawson, Theory of the Nuclear Shell Model (Oxford University Press, Oxford, 1980), Chap. 7.

[28] L. Zamick, D. C. Zheng, S. J. Lee, J. A. Caballero, and E. Moy'a de Guerra, Ann. Phys. (N.Y.) 212, 402 (1991).

[29] H. Bhang et al., Phys. Rev. Lett. 81, 4321 (1998).

[30] J. J. Szymanski et al., Phys. Rev. C 43, 849 (1991).

[31] H. Noumi et al., Phys. Rev. C 52, 2936 (1995).

[32] S. Ajimura et al., Phys. Lett. B 282, 293 (1992).

[33] T. A. Armstrong et al., Phys. Rev. C 47, 1957 (1993).

[34] H. Ohm et al., Phys. Rev. C 55, 3062 (1997). 